\title{
Bioactive components from garlic on brain resiliency against neuroinflammation and neurodegeneration (Review)
}

\author{
HAILONG SONG $^{1}$, JIANKUN CUI ${ }^{1,2}$, VALERI V. MOSSINE ${ }^{3}$, \\ C. MICHAEL GREENLIEF ${ }^{4}, \mathrm{KEVIN} \mathrm{FRITSCHE}^{5}$, GRACE Y. SUN ${ }^{1,3}$ and ZEZONG GU ${ }^{1,2}$ \\ ${ }^{1}$ Department of Pathology and Anatomical Sciences, University of Missouri School of Medicine, \\ Columbia, MO 65212; ${ }^{2}$ Truman VA Hospital Research Service, Columbia, MO 65201; ${ }^{3}$ Department of Biochemistry, \\ University of Missouri School of Medicine, Columbia, MO 65212; ${ }^{4}$ Department of Chemistry, and \\ ${ }^{5}$ Division of Animal Sciences, University of Missouri, Columbia, MO 65211, USA
}

Received July 19, 2019; Accepted September 5, 2019

DOI: $10.3892 /$ etm.2019.8389

\begin{abstract}
Garlic (Allium sativum) has been widely used for culinary and medicinal purposes. Aged garlic extract (AGE) and sulfur-containing compounds, including S-allylcysteine (SAC) are well documented botanical active components of garlic. AGE is prepared by the prolonged extraction of fresh garlic with aqueous ethanol and is considered a nutritional supplement with potential to promote human health. SAC is a water-soluble organosulfur compound and the most abundant component of AGE. Studies have demonstrated that both AGE and SAC can exert neuroprotective effects against neuroinflammation and neurodegeneration. Another bioactive component in AGE is $N$ - $\alpha$-(1-deoxy-D-fructos-1-yl)-L-a rginine (FruArg) although less is known about the metabolic activity of this compound. The main aim of this review was to provide an undated overview of the neuroprotective perspectives of these active garlic components (AGE, SAC and FruArg). Of interest, our studies and those of others indicate that both AGE and FruArg are involved in the regulation of gene transcription and protein expression. AGE has been shown to reverse $67 \%$ of the transcriptome alteration induced by endotoxins-lipopolysaccharide (LPS), and FruArg has been shown to account for the protective effects by reversing $55 \%$ of genes altered in a cell-based neuroinflammation paradigm stimulated by LPS in murine BV-2 microglial cells. AGE and FruArg can alleviate neuroinflammatory responses through a variety of signaling pathways, such as Toll-like receptor and interleukin (IL)-6 signaling, as well as by upregulating the nuclear factor erythroid 2-related factor 2 (Nrf2)-mediated
\end{abstract}

Correspondence to: Dr Zezong Gu, Department of Pathology and Anatomical Sciences, University of Missouri School of Medicine, Columbia, MO 65212, USA

E-mail: guze@health.missouri.edu

Key words: garlic, aged garlic extract, $\mathrm{S}$-allylcysteine, $N$ - $\alpha$-(1-deoxyD-fructos-1-yl)-L-arginine, brain resilience, neuroinflammation, neurodegeneration oxidative stress pathways known to promote microglial resiliency against neuroinflammation and neurodegeneration. The capability of FruArg to pass through the blood-brain barrier further supports its potential as a therapeutic compound. In summary, these experimental results provide new insight into the understanding of the neuroprotective effects of garlic components in promoting brain resiliency for health benefits.

\section{Contents}

1. Introduction

2. Neuroprotective effects of AGE

3. Neuroprotective effects of SAC: A major active component of AGE

4. FruArg: A potential alternative compound with which to mitigate neuroinflammation

5. Conclusions

\section{Introduction}

Garlic (Allium sativum L.) is well-recognized as a botanical resource for health maintenance, largely due to its active components with antioxidant properties (1). However, fresh garlic causes indigestion and its odor is a possible social deterrent (2). Fresh garlic soaked in $15-20 \%$ aqueous ethanol for $>10$ months at room temperature produces aged garlic extract (AGE), which is odorless and contains rich antioxidant chemicals that prevent oxidative damage $(3,4)$. It has been demonstrated that AGE can reduce oxidative damage by scavenging reactive oxygen species (ROS) and inhibiting the formation of lipid peroxides (5). In addition, there is evidence to indicate that AGE can reduce the risk of cardiovascular and cerebrovascular diseases, and possibly inhibit carcinogenesis (6-12). One of the major bioactive components in AGE is S-allylcysteine (SAC), a stable organosulfur compound with a thiol group (13). SAC also possesses antioxidant properties and can neutralize excessive electrophilic species (14).

Apart from SAC, AGE also contains a carbohydrate derivative, $N$ - $\alpha$-(1-deoxy-D-fructos-1-yl)-L-arginine (FruArg). FruArg 
is an Amadori rearrangement product from the amino-carbonyl (Maillard) reaction of AGE, which is a non-enzymatic browning reaction occurring between amino acids and sugars $(15,16)$. FruArg was first isolated from the non-saponin fraction of Korean red ginseng, as a principle antioxidant species with potent effects on lowering blood pressure and improving microcirculation $(17,18)$. The antioxidant potential of FruArg has been demonstrated in an in vitro model of atherosclerosis (19), where FruArg was shown to efficiently block copper(II)-induced low density lipoprotein (LDL) oxidation. Moreover, was demonstrated FruArg that inhibited, in a dose-dependent manner, the release of peroxides by macrophages exposed to oxidized LDL (19). Such activities are attributed to the metal-chelating and peroxide-scavenging capabilities of FruArg. Previous studies by our group also demonstrated the ability of both AGE and FruArg to reduce nitric oxide (NO) production in lipopolysaccharide (LPS)-stimulated murine microglial BV-2 cells in a concentration-dependent manner $(20,21)$. Subsequently, we applied quantitative proteomic analysis by two-dimensional differential in-gel electrophoresis (2D-DIGE) integrated with liquid chromatography tandem mass spectrometry (LC-MS/MS) to profile the proteome patterns and to determine the effects of AGE and FruArg on LPS-stimulated BV-2 cells (20). Furthermore, we investigated gene regulation and associated signaling pathways using the RNA-sequencing (RNA-Seq) approach coupled with machine learning based bioinformatics analysis (21). Importantly, in another study on mice using the sensitive ultra-high performance liquid chromatography-tandem mass spectrometry (UPLC-MS/MS) protocol, we obtained evidence that FruArg can pass through the blood-brain barrier in mice (22). The ability of FruArg to penetrate the blood-brain barrier in subnanomolar concentrations suggests its potential to be studied as a therapeutic compound.

Currently, over 340 publications from PubMed are related to studies on AGE. SAC has also been explored in numerous research articles (approximately 270). However, there are currently only 9 studies available on FruArg (Fig. 1), at least to the best of our knowledge. In this review, we summarize the neuroprotective effects of garlic bioactive components, including AGE, SAC and FruArg, respectively, in three separate sections.

\section{Neuroprotective effects of AGE}

AGE is commercially available and has been widely used in studies investigating its beneficial effects on health $(3,19,23)$. Emerging evidence suggests that AGE attenuates the oxidative damage and neuroinflammation underlying a variety of neurological diseases, such as Alzheimer's disease (AD) as well as other age-related neurodegenerative disorders (14). In earlier studies, AGE was found to protect against cellular damage occurring due to the toxic effects of $\beta$-amyloid peptide (A $\beta$ ) in vitro $(24,25)$. Another study also demonstrated the suppressive effects of AGE on both the A $\beta$ load and the numbers of $\mathrm{A} \beta$ plaques in the brains of amyloid precursor protein (APP) transgenic $(\mathrm{Tg})$ mice as compared to untreated $\mathrm{Tg}$ mice used as controls (26). Mechanistically, AGE can inhibit caspase-3 activation, and can counteract the toxicity of $\mathrm{A} \beta(27,28)$. Additionally, in relation to the pathogenesis of AD, AGE can reduce tau hyperphosphorylation with a decreased activity of

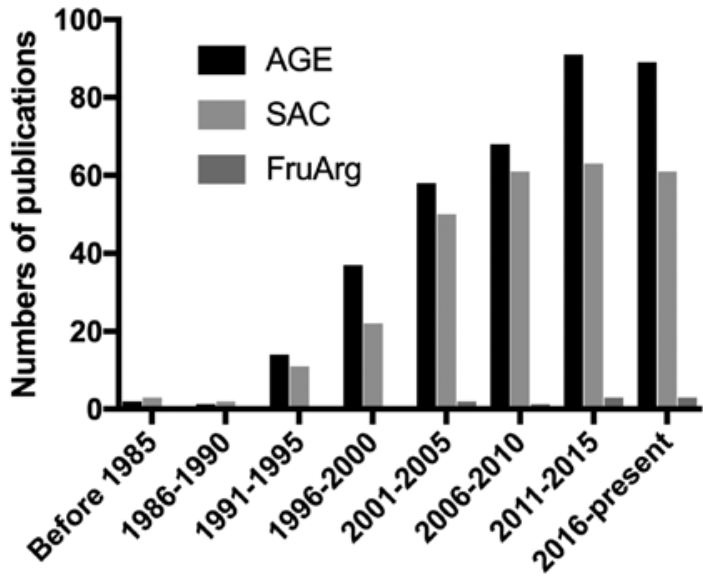

Figure 1. Literature search on 'aged garlic extract (AGE)', 'S-allylcysteine (SAC)' and ' $N$ - $\alpha$-(1-deoxy-D-fructos-1-yl)-L-arginine (FruArg)' from PubMed dated on March 20, 2019.

glycogen synthase kinase (GSK)-3 $\beta$ (26). Furthermore, the neuroprotective effects of AGE on related cognitive dysfunction have also been explored. The oral administration of AGE has been shown to improve spatial recognition memory in cognitively impaired rats induced by $A \beta 42$. The increased expression of synaptic proteins may underlie the improved cognitive functions (29). This effect was also accompanied by the attenuation of the loss of cholinergic neurons and neuroinflammation via the suppression of microglial activation and the secretion of interleukin (IL)-1 $\beta(30,31)$. Neuroinflammation plays an important role in the pathogenesis of a number of neurodegenerative disorders $(32,33)$.

Microglial cells are the resident immune cells in the central nervous system (CNS). After being exposed to brain injuries, stress or infections, microglial cells are the first line of defense that are capable of conferring resilience against oxidative stress and neuroinflammatory responses. Moreover, microglial cells can also become activated and release inflammatory mediators, such as ligands of Toll-like receptors (TLRs), IL-1 $\beta$, tumor necrosis factor- $\alpha$ (TNF- $\alpha)$, reactive oxygen species (ROS) and NO $(34,35)$. A number of botanical compounds with antioxidant properties have been explored to target neuroinflammation. There is evidence to indicate that AGE can exert potent neuroprotective effects via the amelioration of pro-inflammatory responses $(36,37)$.

The effects of AGE on neuroinflammation and oxidative stress have been extensively investigated (38). AGE can reduce the activation of microglia and astrocytes [by immune-detection of the respective ionized calcium-binding adapter molecule 1 (Iba1) and Glial fibrillary acidic protein (GFAP) expression levels], and can thus mitigate neuroinflammatory responses $(13,39)$. Additionally, AGE can reduce the expression levels of neuroinflammatory mediators, including IL-1 $\beta$, nuclear factor $(\mathrm{NF})-\kappa \mathrm{B}$, TLR4, nuclear factor erythroid 2-related factor 2 ( Nrf2) and heme oxygenase 1 (HO-1) in microglial and endothelial cells and/or A $\beta$-induced neuroinflammation in rats $(21,30,40)$. AGE has also been found to alleviate oxidative stress by scavenging ROS, inhibiting LDL oxidation, enhancing antioxidant signaling [such as superoxide dismutase (SOD), glutathione peroxidase (GPX) and glutathione (GSH)] and mitigating lipid peroxidation 


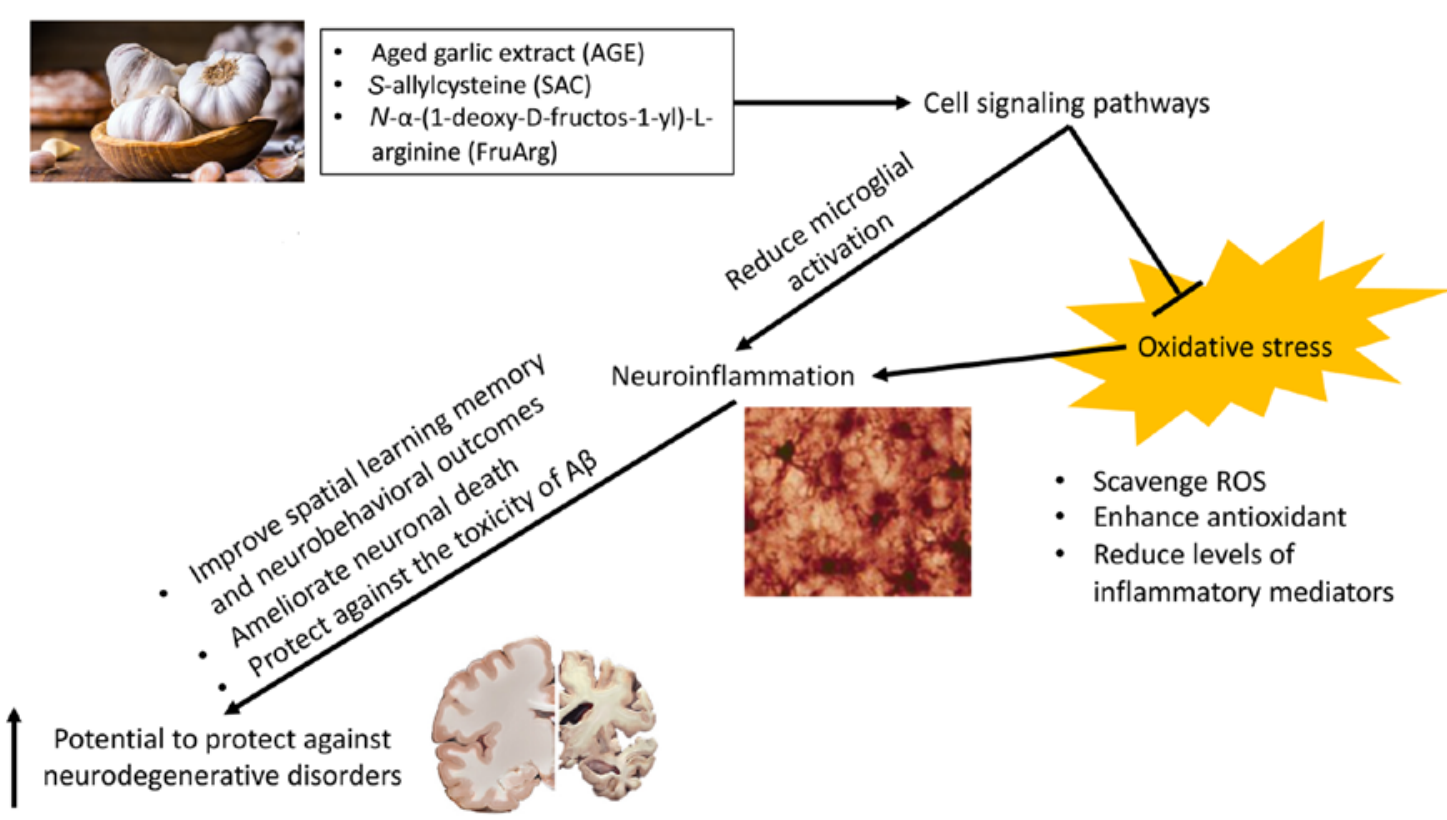

Figure 2. Schematic diagram depicting the neuroprotective effects of garlic components on oxidative stress, neuroinflammation and neurodegeneration. ROS, reactive oxygen species; $\mathrm{RNS}$, reactive nitrogen species; $\mathrm{A} \beta, \beta$ amyloid; $\mathrm{BBB}$, blood-brain barrier.

[malondialdehyde (MDA)] (41-45). Our previous study also demonstrated the protective effects of AGE on ROS and NO production from L-arginine by NO synthase (NOS) (20). Excessive NO can undergo oxidative-reductive reactions and cause cellular damage by endangering reactive nitrogen species (RNS) (46). Nitrosative stress elicited by NO and RNS has been implicated in the pathogenesis of neurodegenerative diseases, such as AD, Parkinson's disease and traumatic brain injury (TBI) (47-52). Previous studies by our group demonstrated that AGE can attenuate NO production in microglial BV-2 cells $(20,21)$. Specifically, treatment with AGE (0.1-1\%) or FruArg $(2-5 \mathrm{mM})$ for $20 \mathrm{~h}$ was found to significantly reduce NO production in a dose-dependent manner in BV2 cells against LPS-induced neuroinflammation (20). Additionally, AGE was found to be capable of regulating both marker protein and gene expression levels, such as caspase-3, IL-6, NF- $\kappa$ B, HO-1, SOD2 and peroxiredoxin-1 (PRDX1), against LPS-induced neuroinflammation (21). By using quantitative proteomics and RNA-Seq analysis, it is possible to unbiasedly identify key canonical pathways associated with LPS stimulation, including TLR signaling, IL-6 signaling, IL-10 signaling, tumor necrosis factor receptor 2 (TNRF2) signaling, 14-3-3-mediated signaling, superoxide radical degradation, pentose phosphate pathway (oxidative branch) and Nrf2-mediated oxidative stress response through AGE treatment $(20,21)$. Our findings are consistent with those of others demonstrating that AGE is capable of reducing the oxidative-nitrosative stress, alleviating neuroinflammation and exerting neuroprotective effects against the pathogenesis of neurodegenerative diseases (37).

\section{Neuroprotective effects of SAC: A major active component of AGE}

SAC is formed by the catabolism of $\gamma$-glutamyl-S-allylcysteine. The antioxidant effects of SAC in the nervous system have been reported (53). Indeed, numerous neuroprotective effects of AGE mentioned above are derived from its multipotent phytochemicals, of which SAC is a major component. The amount of bioactive SAC from AGE increases during the aging process of the garlic, and SAC plays important roles as an antioxidant (37). SAC has been found to destabilize $\mathrm{A} \beta$ fibrils in vitro (54). A $\beta$-induced hippocampal neurodegeneration and neuronal cell death can be prevented by SAC treatment, which is mediated through the caspase-12-dependent pathway and SAC can attenuate endoplasmic reticulum stress (41). SAC can also inhibit apoptosis by regulating caspase-3 activity via phosphoinositide-3-kinase (PI3K), protein kinase B (PKB/Akt) and c-Jun $\mathrm{N}$-terminal kinase (JNK) signaling $(41,53)$. Research has also indicated that SAC can protect against 1-methyl-4-phenylpyridinium (MPP) toxicity associated with reduced lipid peroxidation and superoxide radical production. As a result, SAC is able to improve the MPP-induced locomotor impairment in a mouse model of Parkinson's disease (PD) (55). Researchers have shown the protective effects of SAC on other neurobehavioral outcomes. The oral administration of SAC has been shown to protect against LPS-induced cognitive deficits in rats, and to improve spatial recognition memory in the Y-maze, the discrimination ratio in the novel object discrimination task, and the retention and recall in the passive avoidance test (13). SAC has been shown to alleviate the spatial recognition memory and cognitive deficits associated with novelty recognition and passive avoidance in rats (56). These studies identified the reducing effects of SAC on acetylcholinesterase activity, the lipid peroxidation marker, MDA, as well as its promoting effects on the levels of antioxidant system markers, including SOD, catalase and reduced GSH in the hippocampal brain subregion. Data have also demonstrated that SAC can modulate NF- $\kappa B$, TLR- 4 and TNF- $\alpha$, and prevent the reduction of Nrf2 and HO-1 $(13,56,57)$. Due to its anti-inflammatory properties, a recent study used SAC for the treatment of 
experimental autoimmune encephalomyelitis in mice. That study demonstrated that SAC not only regulated TNF- $\alpha$, IL-17 and matrix metalloprotease 9 levels, but was also capable of attenuating inflammatory cell infiltration, axonal demyelination and axonal loss in the lumbar spinal cord (58). Additionally, another study used allyl sulfide in aging mice and demonstrated that it ameliorated gut dysbiosis and memory functions associated with IncRNA-Hotair regulation and $\mathrm{H} 3 \mathrm{~K} 27 \mathrm{ac}$ regulatory activity (59). Taken together, these neuroprotective effects, namely the reduction of oxidative stress and inflammatory responses may be the key underlying benefits for SAC against neurodegeneration.

\section{FruArg: A potential alternative compound with which to mitigate neuroinflammation}

FruArg, also known as fructosyl arginine, is fractioned from AGE as a bioactive compound and can be chemically synthesized (23). FruArg is identified in AGE at a concentration range of 2.1-2.4 mmol/1. The antioxidant activity of FruArg is as potent as that of ascorbic acid, considering its hydrogen peroxide scavenging activity $(23,42)$. FruArg was first studied, along with AGE and SAC, in the treatment of sickle cell anemia by inhibiting the formation of dense cells in vitro (60). Since the year 2000, researchers have indicated the anti-tumor effects of FruArg through its ability to inhibit cancer cell proliferation and adhesion (61). However, there is limited information available regarding the neuroprotective effects of FruArg. Therefore, to the best of our knowledge, we were the first to have studied the effects of FruArg on LPS-induced inflammatory responses in BV2 microglial cells $(14,20,21)$. Our previous studies demonstrated that FruArg reduced LPS-induced NO production in a concentration-dependent manner $(20,21)$. The ability of FruArg to mitigate neuroinflammation was investigated using quantitative proteomics and RNA-Seq analysis. We demonstrated that FruArg alleviated oxidative stress and neuroinflammatory responses with similar potency as AGE. In fact, both AGE and FruArg shared a large number of regulated protein and gene markers, with distinct profiles in those differentially expressed targets by bioinformatics analyses of the differential levels of the targets in their proteomes and transcriptomes, respectively, affecting the scales of the signaling pathways. Consistently, FruArg was found to alter the Nrf2-mediated oxidative stress response, 14-3-3-mediated signaling, superoxide radical degradation, glutathione biosynthesis, TLR, IL-10, TNRF2 and IL-6 signaling pathways. Of note, several signaling pathways on protein and gene levels regulated by FruArg were also shared with AGE treatment, supporting that FruArg contributed to certain anti-inflammatory effects of AGE. Additionally, we also identified that FruArg treatment triggered the protein-protein interactions associated with immunological disease, and cellular assembly and organization, cellular movement, cell death and survival and cell morphology $(20,21)$.

Apart from an in vitro analysis showing the anti-inflammatory potential of FruArg, researchers have also examined whether FruArg can be delivered to the brain for the treatment of neurological disorders. The blood-brain barrier is known to block $>98 \%$ of all small molecular drug candidates entering the brain, thus causing a major issue for drug therapy development (62). Therefore, it is critical that we previously demonstrated that FruArg could pass through the blood-brain barrier in subnanomolar concentrations in mice (22). Using the UPLC-MS/MS detection protocol, the results demonstrated that, in spite of being highly hydrophilic, FruArg could be absorbed and appear in the blood circulation following an intraperitoneal injection. The pharmacokinetics analysis determined the elimination rate in plasma samples (fraction of FruArg that is removed from the body per unit time) was $6.91 \times 10^{-2} / \mathrm{min}$, with a half-life (t1/2) of $10.03 \mathrm{~min}$, clearance $(\mathrm{Cl})$ of $1.8 \times 10^{-4} \mathrm{l} \cdot \mathrm{min}$, and volume distribution (VD) of $2.63 \times 10^{-3} 1$. Ongoing studies by our research group aim to utilize ribosome profiling (Ribo-Seq) technology by profiling the mRNA activation to analyze such neuroprotective effects of FruArg in the same in vitro model. This could help us to provide a systematic and quantitative method, data profiling and visualization to further elucidate the molecular and genetic mechanisms of the neuroprotective effects of FruArg. Considering that our studies have provided a basis for the anti-inflammatory properties of FruArg, future studies are warranted to include in-depth investigations to examine the mechanisms whereby FruArg can exert its neuroprotective effects, for the prevention and treatment of neurological disorders.

\section{Conclusions}

In conclusion, the neuroprotective effects of garlic components, including AGE, SAC and FruArg on oxidative stress, neuroinflammation and neurodegeneration were discussed herein (Fig. 2). The health benefits of AGE, SAC and FruArg are supported by extensive research on various experimental paradigms. Of importance, our studies demonstrated that FruArg mitigates LPS-induced neuroinflammatory responses and that it is capable of penetrating the blood-brain barrier. This constitutes important information which can provide a promising basis and may open new avenues for promoting brain resilience for health maintenance against neuroinflammation and neurodegeneration from neurological disorders.

\section{Acknowledgements}

Not applicable.

\section{Funding}

This publication was made possible by the funding of the Department of Pathology and Anatomical Sciences research fund at the University of Missouri School of Medicine (to ZG), as well as by grant no. P50AT006273 from the National Center for Complementary and Integrative Health (NCCIH), the Office of Dietary Supplements (ODS), and the National Cancer Institute (NCI). Its contents are solely the responsibility of the authors and do not necessarily represent the official views of the NIEHS, NCCIH, ODS, NCI, or the National Institutes of Health.

\section{Availability of data and materials}

Not applicable. 


\section{Authors' contributions}

HS, JC and ZG wrote the manuscript with significant scientific contributions and concept inputs from VVM, CMG, KF and GYS. All authors have reviewed and approved the current version of the manuscript.

\section{Ethics approval and consent to participate}

Not applicable.

\section{Patient consent for publication}

Not applicable.

\section{Competing interests}

The authors declare that they have no competing interests.

\section{References}

1. Chung LY: The antioxidant properties of garlic compounds: Allyl cysteine, alliin, allicin, and allyl disulfide. J Med Food 9: 205-213, 2006

2. Heber D: The stinking rose: Organosulfur compounds and cancer. Am J Clin Nutr 66: 425-426, 1997.

3. Borek C: Antioxidant health effects of aged garlic extract. J Nutr 131 (Suppl 3): 1010S-1015S, 2001.

4. Kyo E, Uda N, Kasuga S and Itakura Y: Immunomodulatory effects of aged garlic extract. J Nutr 131 (Suppl 3): 1075S-1079S 2001.

5. Morihara N, Hayama M and Fujii H: Aged garlic extract scavenges superoxide radicals. Plant Foods Hum Nutr 66: 17-21, 2011.

6. Borek C: Garlic reduces dementia and heart-disease risk J Nutr 136 (Suppl 3): 810S-812S, 2006.

7. Mathew B and Biju R: Neuroprotective effects of garlic a review. Libyan J Med 3: 23-33, 2008.

8. Katsuki T, Hirata K, Ishikawa H, Matsuura N, Sumi S and Itoh H: Aged garlic extract has chemopreventative effects on 1,2-dimethylhydrazine-induced colon tumors in rats. J Nutr 136 (Suppl 3): 847S-851S, 2006.

9. Tanaka S, Haruma K, Yoshihara M, Kajiyama G, Kira K, Amagase $\mathrm{H}$ and Chayama K: Aged garlic extract has potential suppressive effect on colorectal adenomas in humans. J Nutr 136 (Suppl 3): 821S-826S, 2006.

10. Varshney R and Budoff MJ: Garlic and Heart Disease. J Nutr 146 416S-421S, 2016.

11. Matsumoto S, Nakanishi R, Li D, Alani A, Rezaeian P, Prabhu S, Abraham J, Fahmy MA, Dailing C, Flores F, et al: Aged garlic extract reduces low attenuation plaque in coronary arteries of patients with metabolic syndrome in a prospective randomized double-blind study. J Nutr 146: 427S-432S, 2016.

12. Ried K, Travica N and Sali A: The effect of aged garlic extract on blood pressure and other cardiovascular risk factors in uncontrolled hypertensives: The AGE at Heart trial. Integr Blood Press Control 9: 9-21, 2016

13. Zarezadeh M,Baluchnejadmojarad T, Kiasalari Z, Afshin-Majd S and Roghani M: Garlic active constituent s-allyl cysteine protects against lipopolysaccharide-induced cognitive deficits in the rat: Possible involved mechanisms. Eur J Pharmacol 795: 13-21, 2017.

14. Qu Z, Mossine VV, Cui J, Sun GY and Gu Z: Protective Effects of AGE and Its Components on Neuroinflammation and Neurodegeneration. Neuromolecular Med 18: 474-482, 2016

15. Finot P: The Maillard reaction in food processing, human nutrition and physiology. Birkäuser Verlag, Basel, Switzerland, 1990.

16. Martins SI, Jongen WM and Van Boekel MA: A review of Maillard reaction in food and implications to kinetic modelling. Trends Food Sci Technol 11: 364-373, 2000.

17. Yukinaga M, Yinan Z, Takeshi T, Kenji K and Hiromichi O: Isolation and Physiological Activites of a New Amino Acid Derivative from Korean Red Ginseng. J Ginseng Res 18: 204-211, 1994.
18. Kitao T, Kon K, Nojima K, Takaku T, Maeda N and Okuda H: Effect of components in non-saponin fraction of red ginseng on microcirculation. J Tradit Med (Toyama) 12: 294-295, 1995

19. Ide N, Lau BH, Ryu K, Matsuura H and Itakura Y: Antioxidant effects of fructosyl arginine, a Maillard reaction product in aged garlic extract. J Nutr Biochem 10: 372-376, 1999.

20. Zhou H, Qu Z, Mossine VV, Nknolise DL, Li J, Chen Z, Cheng J, Greenlief CM, Mawhinney TP, Brown PN, et al: Proteomic analysis of the effects of aged garlic extract and its FruArg component on lipopolysaccharide-induced neuroinflammatory response in microglial cells. PLoS One 9: e113531, 2014.

21. Song H, Lu Y, Qu Z, Mossine VV, Martin MB, Hou J, Cui J, Peculis BA, Mawhinney TP, Cheng J, et al: Effects of aged garlic extract and FruArg on gene expression and signaling pathways in lipopolysaccharide-activated microglial cells. Sci Rep 6: 35323 , 2016.

22. Johnson MC, Song H, Cui J, Mossine VV, Gu Z and Greenlief CM: Development of a Method and Validation for the Quantitation of FruArg in Mice Plasma and Brain Tissue Using UPLC-MS/MS ACS Omega 1: 663-668, 2016.

23. Ryu K, Ide N, Matsuura H and Itakura Y: N alpha-(1-deoxy-Dfructos-1-yl)-L-arginine, an antioxidant compound identified in aged garlic extract. J Nutr 131 (Suppl 3): 972S-976S, 2001.

24. Selassie M, Griffin B, Gwebu N and Gwebu ET: Aged garlic extract attenuates the cytotoxicity of beta-amyloid on undifferentiated PC12 cells. In Vitro Cell Dev Biol Anim 35: 369-370, 1999.

25. Peng Q, Buz'Zard AR and Lau BH: Neuroprotective effect of garlic compounds in amyloid-beta peptide-induced apoptosis in vitro. Med Sci Monit 8: BR328-BR337, 2002.

26. Chauhan NB: Effect of aged garlic extract on APP processing and tau phosphorylation in Alzheimer's transgenic model Tg2576. J Ethnopharmacol 108: 385-394, 2006.

27. Jackson R, McNeil B, Taylor C, Holl G, Ruff D and Gwebu ET: Effect of aged garlic extract on caspase-3 activity, in vitro. Nutr Neurosci 5: 287-290, 2002.

28. Marín N, Romero B, Bosch-Morell F, Llansola M, Felipo V, Romá $\mathbf{J}$ and Romero FJ: Beta-amyloid-induced activation of caspase-3 in primary cultures of rat neurons. Mech Ageing Dev 119: 63-67, 2000

29. Ray B, Chauhan NB and Lahiri DK: Oxidative insults to neurons and synapse are prevented by aged garlic extract and S-allyl-L-cysteine treatment in the neuronal culture and APP-Tg mouse model. J Neurochem 117: 388-402, 2011.

30. Nillert N, Pannangrong W, Welbat JU, Chaijaroonkhanarak W, Sripanidkulchai K and Sripanidkulchai B: Neuroprotective effects of aged garlic extract on cognitive dysfunction and neuroinflammation induced by $\beta$-amyloid in rats. Nutrients 9: 9, 2017.

31. Thorajak P, Pannangrong W, Welbat JU, Chaijaroonkhanarak W, Sripanidkulchai K and Sripanidkulchai B: effects of aged garlic extract on cholinergic, glutamatergic and GABAergic systems with regard to cognitive impairment in $A \beta$-induced rats. Nutrients 9: 9, 2017.

32. Gelders G, Baekelandt V and Van der Perren A: Linking Neuroinflammation and Neurodegeneration in Parkinson's Disease. J Immunol Res 2018: 4784268, 2018.

33. Ransohoff RM: How neuroinflammation contributes to neurodegeneration. Science 353: 777-783, 2016.

34. Graeber MB and Streit WJ: Microglia: Biology and pathology. Acta Neuropathol 119: 89-105, 2010.

35. Block ML, Zecca L and Hong JS: Microglia-mediated neurotoxicity: Uncovering the molecular mechanisms. Nat Rev Neurosci 8: 57-69, 2007.

36. Sun AY, Wang Q, Simonyi A and Sun GY: Botanical phenolics and brain health. Neuromolecular Med 10: 259-274, 2008.

37. Colín-González AL, Santana RA, Silva-Islas CA, Chánez-Cárdenas ME, Santamaría A and Maldonado PD: The antioxidant mechanisms underlying the aged garlic extract- and S-allylcysteine-induced protection. Oxid Med Cell Longev 2012: 907162, 2012

38. Ray B, Chauhan NB and Lahiri DK: The 'aged garlic extract': (AGE) and one of its active ingredients S-allyl-L-cysteine (SAC) as potential preventive and therapeutic agents for Alzheimer's disease (AD). Curr Med Chem 18: 3306-3313, 2011.

39. Takechi R, Pallebage-Gamarallage MM, Lam V, Giles C and Mamo JC: Nutraceutical agents with anti-inflammatory properties prevent dietary saturated-fat induced disturbances in blood-brain barrier function in wild-type mice. J Neuroinflammation 10: 73, 2013. 
40. Hiramatsu K, Tsuneyoshi T, Ogawa T and Morihara N: Aged garlic extract enhances heme oxygenase-1 and glutamate-cysteine ligase modifier subunit expression via the nuclear factor erythroid 2-related factor 2-antioxidant response element signaling pathway in human endothelial cells. Nutr Res 36: $143-149,2016$

41. Kosuge Y, Koen Y, Ishige K, Minami K, Urasawa H, Saito H and Ito Y: S-allyl-L-cysteine selectively protects cultured rat hippocampal neurons from amyloid beta-protein- and tunicamycin-induced neuronal death. Neuroscience 122: 885-895, 2003.

42. Ide N and Lau BH: Aged garlic extract attenuates intracellular oxidative stress. Phytomedicine 6: 125-131, 1999.

43. Ide $\mathrm{N}$ and Lau BH: Garlic compounds minimize intracellular oxidative stress and inhibit nuclear factor-kappa $\mathrm{b}$ activation. J Nutr 131 (Suppl 3): 1020S-1026S, 2001.

44. Dillon SA, Burmi RS, Lowe GM, Billington D and Rahman K: Antioxidant properties of aged garlic extract: An in vitro study incorporating human low density lipoprotein. Life Sci 72: $1583-1594,2003$.

45. Thomson M, Al-Qattan KK, Js D and Ali M: Anti-diabetic and anti-oxidant potential of aged garlic extract (AGE) in streptozotocin-induced diabetic rats. BMC Complement Altern Med 16: 17, 2016.

46. Calabrese V, Mancuso C, Calvani M, Rizzarelli E, Butterfield DA and Stella AM: Nitric oxide in the central nervous system: Neuroprotection versus neurotoxicity. Nat Rev Neurosci 8: 766-775, 2007.

47. Pacher P, Beckman JS and Liaudet L: Nitric oxide and peroxynitrite in health and disease. Physiol Rev 87: 315-424, 2007.

48. Raichle ME and Snyder AZ: A default mode of brain function: A brief history of an evolving idea. Neuroimage 37: 1083-1090, discussion 1097-1099, 2007.

49. Giasson BI, Ischiropoulos H, Lee VM and Trojanowski JQ: The relationship between oxidative/nitrative stress and pathological inclusions in Alzheimer's and Parkinson's diseases. Free Radic Biol Med 32: 1264-1275, 2002.

50. Danielson SR and Andersen JK: Oxidative and nitrative protein modifications in Parkinson's disease. Free Radic Biol Med 44 1787-1794, 2008.

51. Besson VC, Croci N, Boulu RG, Plotkine $M$ and Marchand-Verrecchia C: Deleterious poly(ADP-ribose)polymerase-1 pathway activation in traumatic brain injury in rat. Brain Res 989: 58-66, 2003.

52. Besson VC: Drug targets for traumatic brain injury from poly(ADP-ribose)polymerase pathway modulation. Br J Pharmacol 157: 695-704, 2009.
53. Chauhan NB and Mehla J: Ameliorative Effects of Nutraceuticals in Neurological Disorders. In: Bioactive Nutraceuticals and Dietary Supplements in Neurological and Brain Disease. Watson RR and Preedy VR (eds). Academic Press, San Diego, CA, pp245-260, 2015.

54. Gupta VB and Rao KS: Anti-amyloidogenic activity of S-allyl-L-cysteine and its activity to destabilize Alzheimer's beta-amyloid fibrils in vitro. Neurosci Lett 429: 75-80, 2007.

55. Rojas P, Serrano-García N, Medina-Campos ON, Pedraza-Chaverri J, Maldonado PD and Ruiz-Sánchez E: S-Allylcysteine, a garlic compound, protects against oxidative stress in 1-methyl-4-phenylpyridinium-induced parkinsonism in mice. J Nutr Biochem 22: 937-944, 2011.

56. Baluchnejadmojarad T, Kiasalari Z, Afshin-Majd S, Ghasemi Z and Roghani M: S-allyl cysteine ameliorates cognitive deficits in streptozotocin-diabetic rats via suppression of oxidative stress, inflammation, and acetylcholinesterase. Eur J Pharmacol 794: 69-76, 2017.

57. Shi H, Jing X, Wei X, Perez RG, Ren M, Zhang X and Lou H: $\mathrm{S}$-allyl cysteine activates the Nrf2-dependent antioxidant response and protects neurons against ischemic injury in vitro and in vivo. J Neurochem 133: 298-308, 2015.

58. Zeinali H, Baluchnejadmojarad T, Fallah S, Sedighi M, Moradi $\mathrm{N}$ and Roghani M: S-allyl cysteine improves clinical and neuropathological features of experimental autoimmune encephalomyelitis in C57BL/6 mice. Biomed Pharmacother 97: 557-563, 2018.

59. Behera J, Kelly KE and Tyagi N: Altered Non-Coding RNA-Histone Acetylation Regulatory Circuit Is Associated With Cognitive Impairment via Gut Dysbiosis in Aging Mice. The FASEB J 33: 714.3, 2019.

60. Ohnishi ST and Ohnishi T: In vitro effects of aged garlic extract and other nutritional supplements on sickle erythrocytes. J Nutr 131 (Suppl 3): 1085S-1092S, 2001.

61. Mossine VV, Chopra P and Mawhinney TP: Interaction of tomato lycopene and ketosamine against rat prostate tumorigenesis. Cancer Res 68: 4384-4391, 2008.

62. Banks WA: From blood-brain barrier to blood-brain interface: New opportunities for CNS drug delivery. Nat Rev Drug Discov 15: 275-292, 2016

This work is licensed under a Creative Commons Attribution-NonCommercial-NoDerivatives 4.0 International (CC BY-NC-ND 4.0) License. 